\title{
Effectiveness and side effects of immunomodulatory agents for complex regional pain syndrome
}

\author{
Omar Rezk Alshaer ${ }^{1 *}$, Abdullah Obaid Binobaid ${ }^{1}$, Anas Ahmed Nanoh'², Ali Mohammed Asiri², \\ Khalid Saeed Alravie ${ }^{2}$, Abdulhadi Mushabbab Alyahya ${ }^{2}$, Mohammed Ali Alotaif ${ }^{2}$, \\ Nawaf Ahmed Yahya ${ }^{2}$, Zuhair Abshan Alshehri², \\ Anas Mohammed Zarbah², Mohammed Saeed Alqahtani ${ }^{2}$
}

\author{
${ }^{1}$ Department of Internal Medicine, Security Forces Hospital, Riyadh, Saudi Arabia \\ ${ }^{2}$ College of Medicine, King Khalid University, Abha, Saudi Arabia
}

Received: 15 August 2021

Accepted: 20 August 2021

*Correspondence:

Dr. Omar Rezk Alshaer,

E-mail: oalshaer@sfh.med.sa

Copyright: (C) the author(s), publisher and licensee Medip Academy. This is an open-access article distributed under the terms of the Creative Commons Attribution Non-Commercial License, which permits unrestricted non-commercial use, distribution, and reproduction in any medium, provided the original work is properly cited.

\begin{abstract}
The main causes of Complex regional pain syndrome (CRPS) are not fully understood. However, it usually emerges following a surgery or an injury to a limb. Inflammation, musculoskeletal, neuronal, and microvascular abnormalities have been found to contribute to the progression of the disease. Immune cells activation, especially with regards to the early phases of the disease is also indicated in the literature. Accordingly, efforts were directed to manage and enhance such responses, aiming to enhance the outcomes by introducing many immunomodulatory agents for managing patients with CRPS and relieving the associated pain. In this literature review, we have discussed the effectiveness and safety of common immunomodulatory agents for CRPS. We have mainly reviewed the roles of glucocorticoids, TNF- $\alpha$ antagonists, bisphosphonates, and intravenous immunoglobulins administration. Glucocorticoids were among the first reported modalities in the management of the syndrome. However, their use is approached with caution because of their potential systemic side effects. Bisphosphonates and TNF- $\alpha$ antagonists also have validated efficacies and should be administered within the early inflammatory phases to achieve better outcomes. Intravenous immunoglobulins are not adequately validated among the current studies, and evidence shows that they might be useful for a certain subgroup. Therefore, further investigations are required to formulate stronger evidence.
\end{abstract}

Keywords: Complex regional pain syndrome, CRPS, Immunomodulation, Anti-Inflammatory, Management, Efficacy

\section{INTRODUCTION}

Complex regional pain syndrome (CRPS) can be defined as a debilitating type of posttraumatic painful manifestations. The main causes of CRPS are not fully understood. Nevertheless, it usually emerges following a surgery or an injury to a limb. Various symptoms have been reported for patients suffering from CRPS, and are usually inconsistent among them. These symptoms include abnormal sensory manifestations, motor dysfunctions (including dystonia and loss of muscular coordination), autonomic dysfunction (including color and temperature changes to the skin, and vasomotor instability), and atrophic changes (including reduced or increased nail and hair growth, in addition to bone and tissue dystrophy). ${ }^{1,2}$ Some risk factors have also been reported for CRPS, including injured limb immobilization and inciting trauma. ${ }^{3,4}$

Inflammation, musculoskeletal, neuronal, and microvascular abnormalities have been found to contribute to the progression of the disease. ${ }^{5,6}$ Immune cells 
activation, especially with regards to the early phases of the disease is also indicated in the literature. ${ }^{7-9}$ Increased levels of inflammatory markers and subpopulations of Tlymphocytes as CD8+ and CD4+ were also detected by using flow cytometry for sample blood from patients with CRPS, indicating the potential role of the lymphocytic and immune response to the injury. ${ }^{10}$ Accordingly, efforts were directed to manage and enhance such responses, aiming to enhance the outcomes by introducing many immunomodulatory agents for managing patients with CRPS and relieving the associated pain. In the present literature review, we aim to discuss the effectiveness and safety of common immunomodulatory agents for CRPS.

\section{METHODS}

This literature review is based on an extensive literature search in Medline, Cochrane, and EMBASE databases which was performed on 1st August 2021 using the medical subject headings (MeSH) or a combination of all possible related terms. ${ }^{11,12}$ This was followed by the manual search for papers in Google Scholar while the reference lists of the initially included papers. ${ }^{13,14}$ Papers discussing the effectiveness and safety of common immunomodulatory agents for CRPS were screened for relevant information, with no limitation on date, language, age of participants, or publication type.

\section{DISCUSSION}

\section{TNF-a Antagonists}

It has been demonstrated that joint pain is usually developed and maintained through a process that is mediated by the presence of pro-inflammatory cytokines as demonstrated in patients suffering from rheumatological diseases. ${ }^{15}$ Moreover, it has been demonstrated that high levels of tumor necrosis factor- $\alpha$ (TNF- $\alpha$ ), and interleukin6 (IL-6) were observed in an impacted limb of a patient suffering from CRPS, indicating the fact that TNF- $\alpha$ antagonists can be used to effectively manage these patients within the first stages of the disease. ${ }^{16-18}$ This stage can be detected by clinicians by adequately observing the signs of inflammation which are usually predominant during a certain stage when the disease develops. Not many studies have investigated the efficacy of these modalities. However, we tried to collect evidence from all studies in the current literature. Some case reported was published in Europe and the United States indicating the significant effects that were obtained following the administration of intravenous infliximab. ${ }^{19}$ In another context, previous studies have demonstrated that the administration of TNF- $\alpha$ antagonists can be done through subcutaneous routes. ${ }^{20}$ However, this approach is not wellvalidated in patients suffering from CRPS. Previous studies also indicated that thalidomide administration with various doses can significantly have a potent analgesic impact, as it has been reported to induce macrophage recruitment events leading to a significant inhibition on the production of and IL-10 and interferes with TNF mRNA, leading to reduced levels of TNF- $\alpha .^{21}$ Investigations showed that thalidomide was effectively used in multiple myeloma and Behçet's disease patients suffering from CRPS, and another study showed that $31 \%$ of the included cases had a significant improvement following the administration of high doses of thalidomide. ${ }^{22,23}$ Lenalidomide, which is similar to thalidomide but less toxic, was also effectively reported to relieve a wide variety of symptoms in patients suffering from CRPS I, after being treated with other treatment modalities, that even extended for more than 104 weeks in some of the included patients. ${ }^{24}$ However, it should be noted that both of these modalities have been reported with some adverse events, including the potential development of teratogenicity, associated rash, constipation, sedation, and peripheral neuropathy. Further investigations are still needed for further explanation of the efficacy and safety of these modalities in treating patients suffering from CRPS.

\section{Bisphosphonates}

Studies in the literature have demonstrated that these modalities have effective roles in the management of CRPS by different mechanisms that have been adequately discussed. Investigations show that bisphosphonates play a direct role in inhibiting the synthesis and production of IL-6, TNF- $\alpha$, prostaglandin E2, and bradykinin. ${ }^{25}$ Besides, it has been demonstrated that the action of these drugs might also extend to having a direct effect on nociceptors. ${ }^{26,27}$ The indirect action on reducing pain might be comprehended by their ability to improve tissue secondary necrosis, microcirculation of the affected bone, and the increased intramedullary pressure in such events. These abilities are very important within the first phases of the disease where inflammatory markers and inflammation is the hallmark in the pathogenesis of the disease. Many bisphosphonates modalities have been described and validated in the literature. In 1988, pamidronate was first reported as an effective pain-relieving modality with surprising results within three days from the intravenous administration of the treatment modality. ${ }^{26,28}$ Many other investigations have reported the efficacy of other bisphosphonates, including etidronate, alendronate, ibandronate, clodronate, risedronate, and neridronate, however, the duration of action of these modalities is fully comprehended and studied. ${ }^{22}$

Regarding the ability to relieve pain, evidence regarding the validity of using smaller doses of etidronate, alendronate, and risedronate for CRPS than those that are usually used for osteoporosis are comparable, however, the duration is longer and might extend for seven months. ${ }^{29} \mathrm{~A}$ previous randomized controlled trial aimed to validate the efficacy of neridronate and concluded that using bisphosphonates is of many advantages as relieving both the stimulated and spontaneous pain sensations, improving the functional outcomes of patients suffering from acute diseases (that lasted for $<6$ months before initiating the treatment administration), and diseases showing abnormal uptake events as observed on 3-phase scintigraphy, in 
addition to their significant safety profiles that furtherly validated their effectiveness. ${ }^{28,30}$ Another large investigation was also conducted to investigate the efficacy and safety of neridronate and reported that the drug was able to improve and sustain pain sensation in a follow-up duration that lasted for at least one year to their included patients. ${ }^{30}$ It has been demonstrated that a daily dose of 100 $\mathrm{mg}$ of neridronate or $90 \mathrm{mg}$ of pamidronate for a least 10 days can effectively achieve long-term remissions and pain relief. ${ }^{30}$ Furthermore, it should be noted that most of the reported investigations have reported that pain development and maintenance in their recruited populations were associated with osteoclastic overactivities. $^{28,31}$ In addition to their impact on bone metabolism, bisphosphonates also a major role in inducing analgesia by their actions on nociceptors, however, clinical data regarding the validation of such properties are not adequately discussed in the literature. ${ }^{31}$ Accordingly, the efficacy of bisphosphonates might not be restricted to bone-related pain in cases of CRPS and might have other potential effects, however, further studies are still needed. Finally, bisphosphonates can be administered at higher doses for the management of CRPS than those used to manage bone masses loss. It should also be noted that some adverse events as asymptomatic hypocalcemia and flu-like symptoms might be associated with intravenous administration of these modalities. ${ }^{22}$ Accordingly, the administration of these modalities should be cautiously done to prevent any long-term effects on bone metabolism. Besides, further randomized controlled trials are needed for further validation with better recruitment of patients and reporting of the outcomes to adequately validate the results.

\section{Glucocorticoids}

Corticosteroids were among the first modalities that were reported for the management of CRPS, however, the reason behind their use and effectiveness is still controversial among the different studies in the literature. ${ }^{32,33}$ These modalities have been reported to have a direct effect on the inhibition of cyclooxygenase enzymes that are usually found to mediate swelling, pain, and functional impairment. ${ }^{34}$ Besides, research also showed that they have a major anti-inflammatory effect in reducing bone atrophy in patients suffering from CRPS I, reducing the capillary permeability, and reducing the prolonged presence of perivascular infiltrates within the synovium. ${ }^{33,35,36}$ In CRPS I and CRPS II, corticosteroids have also been found to reduce and inhibit ectopic neuronal charges, which might be associated with minor nerve lesions and sensational impairments. ${ }^{37,38}$ Among the variously reported corticosteroids, prednisone remains the commonest that is usually administered at a high oral dose, with a gradual reduction in the dose regimen after the treatment is complete. Studies within the earliest pseudoinflammation stages of CRPS showed that the response rate was estimated to be as high as $82 \% .{ }^{39,40}$ Another investigation also reported that maintaining a dosage regimen of a small dose for three weeks might also enhance the outcomes. ${ }^{40}$ A combination of low-dose dexamethasone and $10 \%$ mannitol was also able to achieve better outcomes regarding pain reduction and improvement in the quality of life. ${ }^{41}$ However, as it is widely known, corticosteroids usually have many systemic adverse effects, can even furtherly impact osteopenia in patients suffering from osteopenic fractures and Sudeck's disease, and can adversely modify mood and memory. ${ }^{42,43}$ Moreover, a previous case of CRPS I has been reported secondary to the administration of corticosteroids. ${ }^{44}$ On the other hand, recent studies have demonstrated that the effectiveness of using corticosteroids has re-emerged because of the recent evidence that shows that corticosteroids can be used for inflammation that occurs proximal to the nerve changes, that are usually similar to CRPS, especially within the early inflammatory phases. ${ }^{45-}$ 47 Finally, further investigations might be needed for adequate evaluation of these modalities, although their long-term administration is not recommended because of their potential impact on the development of systemic complications and adverse events.

\section{Immunoglobulin}

Not many studies have assessed the efficacy of immunoglobulin therapy for the management of CRPS. Early evidence from studies showed that the administration of intravenous immunoglobulins was significantly associated with pain relief in patients suffering from CRPS. ${ }^{48,49}$ On the other hand, a more recent and larger multicenter investigation reported that no significant differences were noticed between the immunoglobulin therapy group and the placebo group in terms of pain relief, indicating that the modality might not have potential effectiveness for patients suffering from CRPS. Therefore, the authors suggested that intravenous immunoglobulin therapy might be potentially beneficial for certain groups of patients. ${ }^{50}$ The mechanism of action of the intravenous immunoglobulins might be attributable to the ability of the administered immunoglobulins to significantly inhibit antibodies from binding to the activating Fc $\gamma$ Rs portions, neutralize the circulating IgG antibodies, upregulate the inhibitory Fc $\gamma$ RIIB portions, or by induction of other methods of immunomodulation. ${ }^{51}$ Aradillas et al also previously indicated that plasma exchange was significantly associated with pain relief in patients suffering from chronic CRPS in their retrospective population. ${ }^{52}$ Previous animal studies have demonstrated that observable enhancements regarding paw edema and mechanical unilateral hyperalgesia were associated with the intraperitoneal administration of IgG antibodies from patients that were previously affected by chronic CRPS to female mice, that was furtherly subjected to unilateral muscle and plantar skin incisions within the injured model limb, as compared to the IgG that was obtained from the healthy controls. ${ }^{53,54}$

\section{CONCLUSION}

In the present literature review, we have discussed the effectiveness and safety of common immunomodulatory agents for CRPS. We have mainly reviewed the roles of glucocorticoids, TNF- $\alpha$ antagonists, bisphosphonates, and intravenous immunoglobulins administration. 
Glucocorticoids were among the first reported modalities in the management of the syndrome. However, their use is approached with caution because of their potential systemic side effects. Bisphosphonates and TNF- $\alpha$ antagonists also have validated efficacies and should be administered within the early inflammatory phases to achieve better outcomes. Intravenous immunoglobulins are not adequately validated among the current studies, and evidence shows that they might be useful for certain subgroups. Therefore, further investigations are required to formulate stronger evidence.

\section{Funding: No funding sources}

Conflict of interest: None declared

Ethical approval: Not required

\section{REFERENCES}

1. Oaklander AL, Horowitz SH. The complex regional pain syndrome. Handb Clin Neurol. 2015;131:481503.

2. $\mathrm{Xu} \mathrm{M}$. Pain and the immune system: emerging concepts of IgG-mediated autoimmune pain and immunotherapies. J Neurol Neurosurg Psychiatry. 2020;91(2):177-88.

3. Allen G, Galer BS, Schwartz L. Epidemiology of complex regional pain syndrome: a retrospective chart review of 134 patients. Pain. 1999;80(3):53944.

4. Terkelsen AJ, Bach FW, Jensen TS. Experimental forearm immobilization in humans induces cold and mechanical hyperalgesia. Anesthesiology. 2008;109(2):297-307.

5. Birklein F. Complex regional pain syndrome phenotypic characteristics and potential biomarkers. Nat Rev Neurol. 2018;14(5):272-84.

6. Gierthmühlen J, Binder A, Baron R. Mechanismbased treatment in complex regional pain syndromes. Nat Rev Neurol. 2014;10(9):518-28.

7. Parkitny L. Inflammation in complex regional pain syndrome: a systematic review and meta-analysis. Neurology. 2013;80(1):106-17.

8. Wei T. Acute versus chronic phase mechanisms in a rat model of CRPS. J Neuroinflammation. 2016;13:14.

9. Lenz M. Local cytokine changes in complex regional pain syndrome type I (CRPS I) resolve after 6 months. Pain. 2013;154(10):2142-9.

10. Russo MA. Expansion and activation of distinct central memory $\mathrm{T}$ lymphocyte subsets in complex regional pain syndrome. $\mathrm{J}$ Neuroinflammation. 2019;16(1):63.

11. Ghozy S. Association of breastfeeding status with risk of autism spectrum disorder: A systematic review, dose-response analysis and meta-analysis. Asian J Psychiatr. 2020;48:101916.

12. Mahmoud AR. Association between sarcoidosis and cardiovascular comorbidity: A systematic review and meta-analysis. Heart Lung. 2020;49(5):512-7.
13. Ghozy S. Therapeutic efficacy of hepatitis B virus vaccine in treatment of chronic HBV infections: A systematic review and meta-analysis. Rev Med Virol. 2020;30(3):e2089.

14. Hashan MR. Association of dengue disease severity and blood group: A systematic review and metaanalysis. Rev Med Virol. 2021;31(1):1-9.

15. Schaible HG. The role of proinflammatory cytokines in the generation and maintenance of joint pain. Ann N Y Acad Sci. 2010;1193:60-9.

16. Huygen FJ. Evidence for local inflammation in complex regional pain syndrome type 1 . Mediators Inflamm. 2002;11(1):47-51.

17. Bernateck M. The first scintigraphic detection of tumor necrosis factor-alpha in patients with complex regional pain syndrome type 1. Anesth Analg. 2010;110(1):211-5.

18. Uçeyler N. Differential expression patterns of cytokines in complex regional pain syndrome. Pain, 2007;132(1-2):195-205.

19. Huygen FJ. Successful treatment of CRPS 1 with anti-TNF. J Pain Symptom Manage. 2004;27(2):1013.

20. Schwartzman S. Does route of administration affect the outcome of TNF antagonist therapy? Arthritis research \& therapy. 2004;6(2):S19-23.

21. Goli V. Does thalidomide have an analgesic effect? Current status and future directions. Curr Pain Headache Rep. 2007;11(2):109-14.

22. Casale RF. Atzeni, and P. Sarzi-Puttini, The therapeutic approach to complex regional pain syndrome: light and shade. Clin Exp Rheumatol. 2015;33(1):126-39.

23. Schwartzman RJ, Chevlen E, Bengtson K. Thalidomide has activity in treating complex regional pain syndrome. Arch Intern Med. 2003;163(12):1487-8.

24. Clark DJ. Autoinflammatory and autoimmune contributions to complex regional pain syndrome. Molecular pain. 2018;14.

25. Strang P. Analgesic effect of bisphosphonates on bone pain in breast cancer patients: a review article. Acta Oncol. 1996;35:50-4.

26. Chauvineau V. [What is the place of diphosphonates in the treatment of complex regional pain syndrome I?]. Ann Readapt Med Phys. 2005;48(3):150-7.

27. Dibas M. Incidence and survival rates and trends of skull Base chondrosarcoma: A Population-Based study. Clin Neurol Neurosurg. 2020;198:106153.

28. Robinson JN, Sandom J, Chapman PT. Efficacy of pamidronate in complex regional pain syndrome type I. Pain Med. 2004;5(3):276-80.

29. Fujita T. Comparison of the analgesic effects of bisphosphonates: etidronate, alendronate and risedronate by electroalgometry utilizing the fall of skin impedance. J Bone Miner Metab. 2009;27(2):234-9.

30. Varenna M. Treatment of complex regional pain syndrome type I with neridronate: a randomized, 
double-blind, placebo-controlled study. Rheumatol. 2013;52(3):534-42.

31. Yanow JM, Pappagallo, Pillai L. Complex regional pain syndrome (CRPS/RSD) and neuropathic pain: role of intravenous bisphosphonates as analgesics. ScientificWorldJournal. 2008;8:229-36.

32. Oyen WJG. Reflex sympathetic dystrophy of the hand: an excessive inflammatory response? Pain, 1993;55(2):151-7.

33. van der Laan L, Goris RJ. Reflex sympathetic dystrophy. An exaggerated regional inflammatory response? Hand Clin. 1997;13(3):373-85.

34. Masferrer JL. Selective regulation of cellular cyclooxygenase by dexamethasone and endotoxin in mice. J Clin Invest. 1990;86(4):1375-9.

35. Kozin F. The reflex sympathetic dystrophy syndrome. I. Clinical and histologic studies: evidence for bilaterality, response to corticosteroids and articular involvement. Am J Med. 1976;60(3):32131.

36. Okudan B, Celik C. Determination of inflammation of reflex sympathetic dystrophy at early stages with Tc-99m HIG scintigraphy: preliminary results. Rheumatol Int. 2006;26(5):404-8.

37. Devor M. Govrin-Lippmann R, Raber P. Corticosteroids suppress ectopic neural discharge originating in experimental neuromas. Pain. 1985;22(2):127-37.

38. Dellon LE. Andonian, and G.D. Rosson, Lower extremity complex regional pain syndrome: longterm outcome after surgical treatment of peripheral pain generators. J Foot Ankle Surg. 2010;49(1):33-6.

39. Kozin F. The reflex sympathetic dystrophy syndrome (RSDS). III. Scintigraphic studies, further evidence for the therapeutic efficacy of systemic corticosteroids, and proposed diagnostic criteria. Am J Med. 1981;70(1):23-30.

40. Christense K, Jensen EM, Noer I. The reflex dystrophy syndrome response to treatment with systemic corticosteroids. Acta Chir Scand. 1982;148(8):653-5.

41. Zyluk A, Puchalski P. Treatment of early complex regional pain syndrome type 1 by a combination of mannitol and dexamethasone. J Hand Surg Eur. 2008;33(2):130-6.

42. Bianchi C. Long-term functional outcome measures in corticosteroid-treated complex regional pain syndrome. Eura Medicophys. 2006;42(2):103-11.

43. Brown ES. Effects of glucocorticoids on mood, memory, and the hippocampus. Treatment and preventive therapy. Ann N Y Acad Sci. 2009;1179:41-55.

44. Murphy AD, Lloyd-Hughes H, Ahmed J. Complex regional pain syndrome (Type 1) following steroid injection for stenosing tenosynovitis. J Plast Reconstr Aesthet Surg. 2010;63(10):e740-1.

45. Kingery WS. A critical review of controlled clinical trials for peripheral neuropathic pain and complex regional pain syndromes. Pain. 1997;73(2):123-39.

46. Bove GM. Focal nerve inflammation induces neuronal signs consistent with symptoms of early complex regional pain syndromes. Exp Neurol. 2009;219(1):223-7.

47. Jänig W. The fascination of complex regional pain syndrome. Exp Neurol. 2010;221(1):1-4.

48. Goebel A. Intravenous immunoglobulin treatment of the complex regional pain syndrome: a randomized trial. Ann Intern Med. 2010;152(3):152-8.

49. Goebel A. Human pooled immunoglobulin in the treatment of chronic pain syndromes. Pain Med. 2002;3(2):119-27.

50. Goebel A. Low-Dose Intravenous Immunoglobulin Treatment for Long-Standing Complex Regional Pain Syndrome: A Randomized Trial. Ann Intern Me., 2017;167(7):476-83.

51. Lewis BJB, Branch DR. Mouse Models of Rheumatoid Arthritis for Studies on Immunopathogenesis and Preclinical Testing of $\mathrm{Fc}$ Receptor-Targeting Biologics. Pharmacol. 2020;105(11-12):618-29.

52. Aradillas E. Plasma Exchange Therapy in Patients with Complex Regional Pain Syndrome. Pain Physician. 2015;18(4):383-94.

53. Helyes Z. Transfer of complex regional pain syndrome to mice via human autoantibodies is mediated by interleukin-1-induced mechanisms. Proc Natl Acad Sci. 2019;116(26):13067-76.

54. Tékus V. A CRPS-IgG-transfer-trauma model reproducing inflammatory and positive sensory signs associated with complex regional pain syndrome. Pain. 2014;155(2):299-308.

Cite this article as: Alshaer OR, Binobaid AO, Nanoh AA, Asiri AM, Alravie KS, Alyahya AM et al. Effectiveness and side effects of immunomodulatory agents for complex regional pain syndrome. Int $\mathbf{J}$ Community Med Public Health 2021;8:4627-31. 